\title{
PRIMERA CITA DE CULEX INTERROGATOR (DIPTERA: CULICIDAE) PARA LA HISPANIOLA Y ACTUALIZACIÓN DEL LISTADO DE MOSQUITOS DE JARABACOA, REPÚBLICA DOMINICANA
}

\section{First record of Culex interrogator (Diptera: Culicidae) in Hispaniola and updated checklist of mosquitoes of Jarabacoa, Dominican Republic}

\author{
María Altagracia Rodríguez Sosa ${ }^{1 \mathrm{a}}$, Juan Rueda ${ }^{2}$, Roddy Jesús Pichardo Rodríguez ${ }^{1 \mathrm{~b}}$, \\ Yohan Enmanuel Vásquez Bautista ${ }^{1 c}$, Jesús Confesor Durán Tiburcio ${ }^{1 \mathrm{~d}}$, \\ Rigoberto Fimia-Duarte ${ }^{3}$ y Pedro María Alarcón-Elbal ${ }^{1 \mathrm{e}, *}$
}

\begin{abstract}
${ }^{1}$ Laboratorio de Entomología, Universidad Agroforestal Fernando Arturo de Meriño (UAFAM), Jarabacoa, República Dominicana; ${ }^{1 a}$ (D) orcid.org/0000-0003-3497-6557; ${ }^{\text {b }}$ (iD) orcid.org/0000-0001-9440-5006; ${ }^{\text {lc }}$ (iD) orcid.org/0000-00028989-5757; 1d Dorcid.org/0000-0001-5416-7250; 1e, (D) orcid.org/0000-0001-5319-4257. ${ }^{2}$ Instituto Cavanilles de Biodiversidad y Biología Evolutiva, Universidad de València, Paterna, València, España; (D) orcid.org/0000-00027629-8881. ${ }^{3}$ Facultad de Tecnología de la Salud Julio Trigo López, Universidad de Ciencias Médicas de Villa Clara, Cuba; (D) orcid.org/0000-0001-5237-0810.*Para correspondencia: pedro.alarcon@uv.es.
\end{abstract}

\section{RESUMEN}

Desde que dio inicio el nuevo siglo, varios estudios sobre la culicidofauna de la Hispaniola han elevado el conocimiento de este grupo de artrópodos, muy relevantes desde el punto de vista médico y veterinario. En relación a la diversidad de la familia Culicidae, los hallazgos más recientes se han realizado en el municipio de Jarabacoa, República Dominicana. Con el objetivo de profundizar en la diversidad de los culícidos presentes en esta localidad montañosa de El Cibao, se procedió a la búsqueda de criaderos de mosquitos, sobre todo de origen natural, desde enero de 2018 hasta junio de 2019. La identificación de los especímenes se realizó mediante la utilización de lupa binocular y microscopio, con la ayuda de claves taxonómicas. Se reportan seis nuevas especies para Jarabacoa, sumando un total de 22 para el municipio, sobre las cuales se analizan cuestiones como su bioecología y su papel en la transmisión de agentes patógenos, entre otras. Destaca la presencia de Culex interrogator Dyar y Knab, 1906, especie que se reporta por primera vez en la Hispaniola, y por segunda en el Caribe insular. A pesar de que este culícido no está implicado hasta la fecha en la transmisión de patógenos al ser humano, otras especies encontradas en este estudio como Anopheles crucians Wiedemann, 1828, Aedes scapularis (Rondani, 1848) y Psorophora confinnis (Lynch Arribálzaga, 1891) deben ser tenidas en cuenta en los programas de vigilancia y control, a tenor de su relevancia médica.

Palabras clave: mosquitos, diversidad, vector de enfermedades, República Dominicana, Hispaniola.

\section{ABSTRACT}

Since the beginning of the new century, several studies on the culicid fauna of Hispaniola have increased the knowledge of this group of arthropods, very relevant from the medical and veterinary point of view. Regarding the diversity of the Culicidae family, the most recent findings have been made in the municipality of Jarabacoa, Dominican Republic. In order to deepen the diversity of the culicids in this mountainous locality of El Cibao, a survey of mosquito breeding sites, mainly natural, was conducted from January 2018 to June 2019. The identification of the specimens was carried out using magnifying glass and binocular microscope, with the help of taxonomic keys. Six new species are reported for Jarabacoa, on which questions such as their bioecology and their role in the transmission of pathogens, among others, are analyzed. 
The presence of Culex interrogator Dyar and Knab, 1906, a mosquito species recorded for the first time from Hispaniola, and for the second time in the insular Caribbean, is noteworthy. Although this culicid has not been implicated in the transmission of pathogens to humans to date, others species found in this study such as Anopheles crucians Wiedemann, 1828, Aedes scapularis (Rondani, 1848) and Psorophora confinnis (Lynch Arribálzaga, 1891) should be taken into account in surveillance and control programmes, in view of their medical relevance.

Keywords: mosquitoes, diversity, disease vector, Dominican Republic, Hispaniola.

\section{INTRODUCCIÓN}

Desde que dio inicio el nuevo siglo, varios trabajos sobre la culicidofauna de la Hispaniola han elevado el conocimiento de este grupo de artrópodos, muy relevantes desde el punto de vista médico y veterinario. Peña y Chadee (2004) realizaron un importante aporte a la diversidad, reportando por primera vez a Mansonia dyari Belkin, Heinemann y Page, 1970, Mansonia flaveola (Coquillett, 1906) y Aedeomyia squamipennis (Lynch Arribálzaga, 1878) en República Dominicana. Por su parte, Marquetti Fernández et al. (2012) reportan la presencia de Aedes albopictus (Skuse, 1894) por primera vez para Haití, un culícido invasor de gran relevancia vectorial.

Recientemente, diversas aportaciones han revitalizado los estudios sobre culícidos en el campo de la biodiversidad: Rodríguez-Sosa et al. (2018) encontraron la especie Uranotaenia cooki Root, 1937 por vez primera en República Dominicana; Rueda et al. (2018) hallaron Culex garciai González Broche, 2000, por primera vez en la Hispaniola y Rodríguez Sosa et al. (2019a) informaron de la presencia de Culex biscaynensis Zavortink y O'Meara, 1999, por primera vez en el Caribe insular. De estos últimos hallazgos, todos realizados en el municipio cibaeño de Jarabacoa, se deduce que la diversidad de mosquitos de la isla puede estar todavía bastante subestimada.

Durante 2017, Rodríguez Sosa et al. (2019a) capturaron en Jarabacoa 16 especies de culícidos, pertenecientes a las tres subfamilias conocidas (Anophelinae, Culicinae y Toxorhynchitinae), agrupadas en seis géneros, lo cual supone, aproximadamente, un tercio del total de especies citadas en República Dominicana. De entre estas especies, cinco han sido reportadas como vectores de patógenos a los humanos: Anopheles albimanus Wiedemann, 1820, Culex nigripalpus Theobald, 1901, Culex quinquefasciatus Say, 1823, Aedes aeypti (Linnaeus, 1762) y Ae. albopictus. Otras también deben ser tenidas en cuenta, a pesar de no tener ningún rol aparente en la transmisión de patógenos, al causar considerables molestias producto de sus picaduras.

\section{OBJETIVOS}

- Profundizar en la diversidad de los culícidos de Jarabacoa, complementando el trabajo realizado por Rodríguez Sosa et al. (2019a).

\section{MATERIALES Y MÉTODOS}

Área de estudio. El estudio se realizó en el municipio de Jarabacoa, provincia de La Vega, ubicado en la Región Norte o Cibao de República Dominicana, país que ocupa algo más de los dos tercios orientales de la isla de la Hispaniola, en el archipiélago de las Antillas Mayores. Este municipio, considerado como uno de los mejores destinos caribeños para el ecoturismo, posee un área de $23 \mathrm{~km}^{2}$ y una altura promedio de $530 \mathrm{~m}$ s.n.m. Además, cuenta con numerosas cuencas hidrográficas de gran importancia ecológica asociadas al pico con mayor altitud de todo el Caribe insular, conocido como Pico Duarte (Orgaz Agüera y Cañero-Morales, 2015). 
Captura e identificación de los especímenes. Se procedió a la búsqueda de criaderos de mosquitos, con énfasis en aquellos de origen natural, en diferentes zonas seleccionadas desde enero de 2018 hasta junio de 2019. El material biológico se capturó mediante la técnica del dipping, con la ayuda de pipetas Pasteur y bandejas plásticas para la visualización y separación de los ejemplares preimaginales, los cuales se introdujeron en botes plásticos de cierre hermético, debidamente etiquetados, para su traslado al laboratorio. La periodicidad de los muestreos fue semanal, siempre que las condiciones meteorológicas lo permitieron.

En cuanto al manejo de los individuos en el laboratorio, las muestras se introdujeron en botes de eclosión para el desarrollo de las larvas hasta alcanzar el estadio L4, tras lo cual se procedió a su fijación en alcohol al $70 \%$, previa muerte por inmersión en agua a $60{ }^{\circ} \mathrm{C}$. En el caso de las pupas capturadas, se dejaron emerger los adultos y después se sacrificaron en frío. La identificación se realizó con la ayuda de claves taxonómicas (Darsie y Ward, 2005; González Broche, 2006) y la utilización de lupa binocular y/o microscopio, realizando preparaciones microscópicas con líquido de Hoyer como medio de montaje, cuando fue necesario.

Se ha seguido la clasificación taxonómica proporcionada por el Systematic Catalog of Culicidae de la Walter Reed Biosystematics Unit (Gaffigan et al., 2015). El material biológico se encuentra depositado en la Colección del Laboratorio de Entomología de la Universidad Agroforestal Fernando Arturo de Meriño (UAFAM) de Jarabacoa, República Dominicana.

\section{RESULTADOS Y DISCUSIÓN}

Se capturaron seis nuevas especies de culícidos en relación a los resultados mostrados por Rodríguez Sosa et al. (2019a), agrupados en cuatro tribus, cinco géneros y cinco subgéneros. De todas ellas, Culex (Culex) interrogator Dyar y Knab, 1906, supone el primer registro para la Hispaniola, y el segundo para el Caribe insular.

A continuación, se enlistan los nuevos taxa encontrados, incluyendo apuntes de interés sobre su distribución mundial, datos de captura, características bioecológicas, importancia sanitaria y detalles de su morfología diferencial.

\section{Tribu Anophelini Anopheles (Anopheles) crucians Wiedemann, 1828}

Distribución. Bahamas, Belice, Costa Rica, Cuba, El Salvador, EE. UU., Guatemala, Haití, Honduras, Jamaica, México, Nicaragua, Puerto Rico y República Dominicana (WRBU, 2020).

Capturas. La Joya (196'49.04” N 70³7’8.85” O, 535 m s.n.m.), 20-II-2018, dos larvas; La Trinchera (196'46.34” N 70³7'46.37' O, 560 m s.n.m), 29-VI-2018, cuatro larvas; Pinar Quemado (196'28.57” N 70³8'59.93”' O, 541 m s.n.m.), 09-II-2019, una larva.

Hábitat. Los estados preimaginales se desarrollan en charcos permanentes y/o temporales de agua dulce, pozos, arroyos, pantanos y en los márgenes de lagos, generalmente asociados a la vegetación acuática (Carpenter y LaCasse, 1955). En Jarabacoa, esta especie se ha encontrado en zonas rurales, habitando una laguna permanente en las proximidades del río Baiguate en cohabitación con Culex atratus Theobald, 1901, y en una charca temporal junto a Aedes scapularis (Rondani, 1848), Psorophora confinnis (Lynch Arribálzaga, 1891), Cx. nigripalpus, Ur. cooki, An. albimanus y Cx. atratus; además, se encontró en una zona semiurbana en un neumático usado junto a Anopheles grabhamii Theobald, 1901. 
Importancia médica. Las hembras se alimentan de la sangre del ser humano, aunque prefieren hacerlo de la de animales domésticos (Carpenter y LaCasse, 1955). Se ha demostrado experimentalmente la susceptibilidad de esta especie a Plasmodium vivax (Grassi y Feletti, 1890) y P. falciparum (Welch, 1897), (Haemosporida: Plasmodiidae; Mayne, 1919), y, además, se ha encontrado infectada en la naturaleza, por lo que podría actuar como vector de la malaria (Walter Reed Biosystematics Unit [WRBU], 2020), si bien se considera que An. albimanus es el principal vector en el Caribe (Faran, 1980). Se han encontrado ejemplares positivos a ARN de virus West Nile (WNV) en EE. UU., aunque no se han realizado estudios de competencia vectorial al respecto (Unlu et al., 2010).

Identificación. Es la tercera especie de Anopheles detectada en el municipio y se diferencia morfológicamente de la más cercana, An. grabhamii, por poseer la seda 1-P simple y aislada de la 2-P (González Broche, 2006).

\section{Tribu Culicini Culex (Culex) interrogator Dyar et Knab, 1906}

Distribución. Belice, Costa Rica, Cuba, El Salvador, EE. UU., Guatemala, Honduras, México, Nicaragua, Panamá y Perú (Pérez Menzies et al., 2018; WRBU, 2020). Constituye el primer reporte del culicino para la Hispaniola, y el segundo para el Caribe insular.

Capturas. La Javilla (197'44.94” N 70³8'7.31” O, 525 m s.n.m.), 28-II-2019, 11 larvas; La Javilla (197'44.94” N 70³8'7.31’O, 525 m s.n.m.), 15-III-2019, cinco larvas; La Javilla (197'44.94” N 70³8'7.31” O, 525 m s.n.m.), 25-III-2019, tres larvas; Pinar Quemado (196'28.84" N 70³9'1.31" O, 541 m s.n.m.), 08-VI-2019, una larva; Pinar Quemado (196’27.55” N 70³9’2.08” O, 543 m s.n.m.), 15-VI-2019, seis larvas.

Hábitat. Sus larvas se han encontrado en desagües de aguas pluviales con restos de hierba, zanjas laterales de carreteras y ovitrampas próximas a tierras dedicadas a la agricultura (Shin et al., 2016); en cubetas, bebederos, llantas, floreros, contenedores artificiales, pozos y charcos (Elizondo-Quiroga, 2002; Zapata-Peniche et al., 2007; Ortega-Morales et al., 2010); en fosas sépticas, sótanos inundados, lagunas de oxidación, botellas de plástico y cañadas (Pérez Menzies et al., 2018). En Jarabacoa, se localizó en un tramo remansado de un pequeño arroyo en una zona deprimida, con espesa cobertura arbórea y sombría (Fig. 1, A y B) y en unos charcos de lluvia en una zona de pasto con mucha insolación (Fig. 1, B y C), a una altitud de 537 y 529 m s.n.m., respectivamente, en cohabitación con Culex corniger Theobald, 1903, $C x$. quinquefasciatus y Cx. nigripalpus. La presente aportación aumenta su altitud de detección en más de 100 m s.n.m.

Importancia médica. Se trata de un mosquito ornitofílico, pero también puede alimentarse de la sangre de mamíferos, por consiguiente, podría tener un papel relevante en la transmisión de virus aviares (Cruz Francisco et al., 2012).

Identificación. Las larvas se identifican con facilidad gracias a las características morfológicas del sifón (Fig. 2, A y E) que, además de ser relativamente corto, con un índice sifonal cercano a 2.5, posee un pecten formado por espinas distales (Fig. 2C) mucho mayores que las basales (Fig. 2F) que, en su totalidad, se extienden hasta tres cuartas partes de la longitud del sifón y tienen forma alargada y robusta, siendo este uno de los caracteres distintivos de la especie (Darsie y Ward, 2005). Además, su antena es espiculada (Fig. 2B), las sedas cefálicas C-4 son simples y las C-5 y C-6 ramificadas (Pérez Menzies et al., 2018) (Fig. 2G). Las escamas del segmento VIII poseen flecos, nunca espinas (Fig. 2D). 


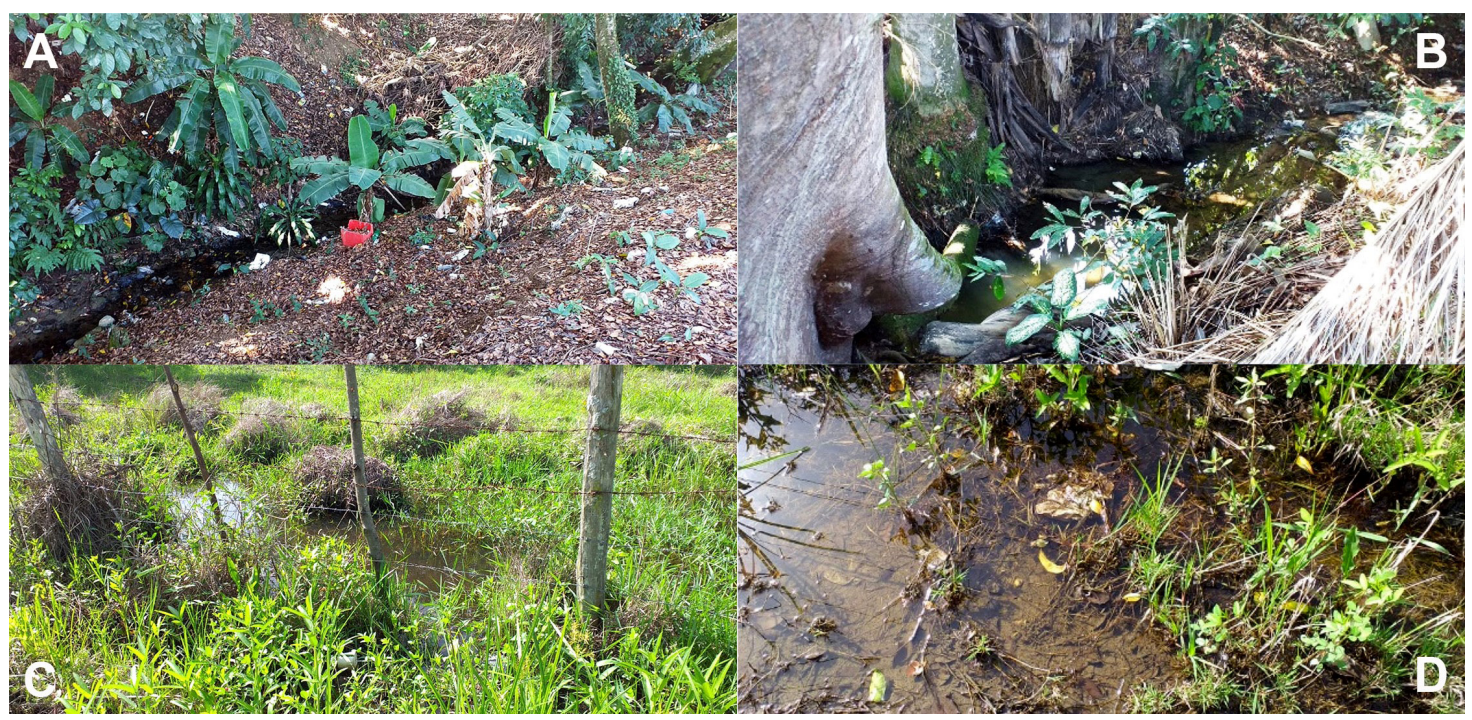

Figura 1. Criaderos de Culex interrogator en Jarabacoa, República Dominicana. A, arroyo. B, detalle de zona remansada. C, charco de agua de lluvia. D, detalle del charco.
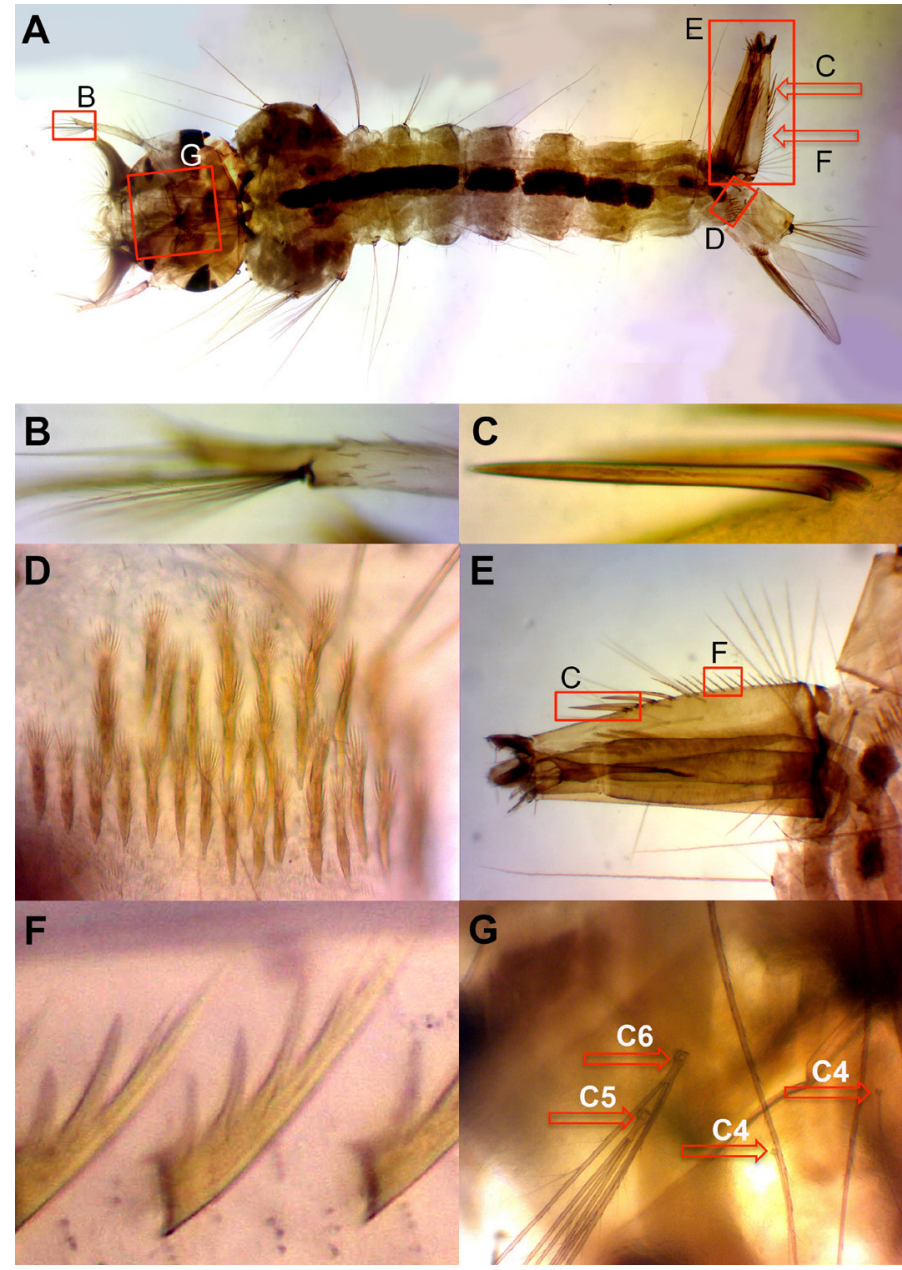

Figura 2. Larva de Culex interrogator. A, vista dorsal. B, antena, seta 1-A. C, espina sifónica distal. D, vista lateral del segmento VIII, espículas del pecten. E, vista lateral del sifón. F, espinas sifónicas basales. G, sedas cefálicas (C-4, C-5 y C-6). 


\section{Tribu Aedini \\ Aedes (Ochlerotatus) scapularis (Rondani, 1848)}

Distribución. Argentina, Bahamas, Colombia, Cuba, Ecuador, EE. UU., Guatemala, Guyana, Haití, Honduras, Jamaica, México, Nicaragua, Panamá, República Dominicana, Trinidad y Tobago y Venezuela (WRBU, 2020).

Capturas. Pinar Quemado (196'28.57'N 70³8'59.93”O, 541 m s.n.m.), 09-II-2019, 11 larvas; Pinar Quemado (196'29.03'N 70³9'0.15'O, 541 m s.n.m.), 06-VI-2019, 61 larvas y cuatro pupas.

Hábitat. Puede habitar ecotonos rurales y urbanos, e incluso comportarse como sinantrópica, estando presente en parques urbanos e introduciéndose en las viviendas para alimentarse (Klein et al., 1992), aspecto ratificado por nuestro equipo de investigación. Las larvas se desarrollan preferentemente en lagunas temporales de origen natural, aunque también pueden explotar contenedores de agua naturales permanentes o artificiales (Forattini et al., 1997). En Jarabacoa, los inmaduros fueron capturados en una zona rural, en charcos temporales ocasionados por la lluvia, junto a Ps. confinnis, Cx. nigripalpus, Ur. cooki, An. albimanus, An. crucians y Cx. atratus. Hemos constatado su marcada antropofilia verificando la presencia de hembras, que poseen un tórax muy característico a simple vista, con un parche grande de escamas blancas ocupando la mitad anterior, alimentándose sobre personas al aire libre, incluso próximas al centro urbano.

Importancia médica. Este mosquito está incriminado en la transmisión de los nematodos Dirofilaria immitis (Leidy, 1856), (Lourenço-de-Oliveira y Deane, 1995) y Wuchereria bancrofti (Cobbold, 1877), (Spirurida: Onchocercidae; Rachou et al., 1995), y de él se han aislado al menos 15 virus, incluyendo el de la fiebre amarilla (YFV), (Arnell, 1976), encefalitis equina venezolana (VEEV), (Mitchell et al., 1985), Rocío (Mitchell y Forattini, 1984), Ilheus (Pauvolid-Corrêa et al., 2013) y Melao (Spence et al., 1962). Ha sido recientemente reportado como portador de huevos de Dermatobia hominis (Linnaeus, 1781) (Diptera: Oestridae), un braquícero parásito que causa miasis en el ser humano, en el sur de Brasil (Marchi et al., 2012).

Identificación. A diferencia del resto de Aedes capturados en el municipio, Aedes albonotatus (Coquillett, 1905), Ae. albopictus y Ae. aegypti, las escamas del segmento VIII se distribuyen en varias hileras; además, su tórax y abdomen están cubiertos espesamente con espículas muy visibles y la seda protorácica 7-P posee tres ramas (González Broche, 2006).

\section{Psorophora (Grabhamia) confinnis (Lynch Arribálzaga, 1891)}

Distribución. Argentina, Bahamas, Belice, Bolivia, Brasil, Colombia, Costa Rica, Cuba, El Salvador, EE. UU., Guatemala, Guyana, Guyana Francesa, Haití, Honduras, Islas Caimán, Islas Vírgenes, México, Nicaragua, Panamá, Paraguay, Perú, Puerto Rico, República Dominicana, Uruguay y Venezuela (WRBU, 2020). En República Dominicana se encontraba citada con el sinónimo de Psorophora jamaicensis (Theobald, 1901).

Capturas. Pinar Quemado (196’28.84’N 70³9’1.31'O, 541 m s.n.m.), 06-VI-2019, dos larvas.

Hábitat. Sus estados preimaginales se desarrollan en charcos temporales de agua de lluvia, con vegetación o sin ella, zonas encharcadas frecuentadas por animales de granja y en huellas de ganado (Belkin y Heinemann, 1973; González Broche, 2006). En Jarabacoa, esta especie fue capturada puntualmente en una zona rural en un charco temporal de lluvia, con abundante vegetación circundante y en cohabitación con Cx. nigripalpus y Ae. scapularis. Hemos constatado su marcada antropofilia verificando la presencia de hembras alimentándose sobre nosotros en las inmediaciones de su hábitat. 
Importancia médica. Las hembras pueden alimentarse de sangre humana, aunque muestran preferencia por la del ganado vacuno (González Broche, 2006). Debido a su alta susceptibilidad, su abundancia en regiones enzoóticas y epizoóticas y sus preferencias alimentarias, este aedino está considerado como un importante vector puente para el VEEV enzoótico y epizoótico (Ortiz et al., 2005).

Identificación. Las características principales del subgénero Grabhamia Theobald, 1903, se centran, por un lado, en que posee menos de 10 espículas en el pecten sifonal y, por otro, que el tamaño de sus antenas no supera el largo de la cabeza (González Broche, 2006). Además, según el mismo autor, la sedas 5-C y 6-C son ramificadas y las espículas del pecten sifonal son estrechas y con 1-2 espinas laterales cortas.

\section{Psorophora (Grabhamia) infinis (Dyar y Knab, 1906)}

Distribución. Cuba, Ecuador, EE. UU., Haití, Jamaica, México y República Dominicana (WRBU, 2020).

Capturas. La Confluencia (199'8.56” N 70³8'34.48” O, 478 m s.n.m.), 06-XI-2018, 11 larvas; La Confluencia (199'8.58' N 70³8’40.30” O, 477 m s.n.m.), 16-XI-2018, cinco larvas y una pupa.

Hábitat. La especie cría en huecos de rocas, cangrejeras, charcos de agua de lluvia, zanjas y áreas encharcadas frecuentadas por animales de granja, además de tanques, cisternas y canales de concreto para drenaje (Belkin et al., 1970; Belkin y Heinemann, 1973). En Jarabacoa, sus larvas fueron capturadas en una zona semiurbana, en rodadas y residuos sólidos urbanos de poliestireno, en las proximidades de la confluencia entre los ríos Jimenoa y Yaque del Norte.

Importancia médica. No se le conoce implicación en la transmisión de entidades infecciosas.

Identificación. A diferencia de Ps. confinnis, posee las sedas de la cabeza 6-C simples y las espículas del pecten sifonal son anchas y con 3-5 espinas laterales también largas (González Broche, 2006).

\section{Tribu Sabethini}

\section{Wyeomyia (Wyeomyia) vanduzeei Dyar et Knab, 1906}

Distribución. Cuba, EE. UU., Haití, Islas Caimán, Jamaica, Puerto Rico y República Dominicana (WRBU, 2020). En República Dominicana se encontraba citada con el sinónimo de Wyeomyia sororcula Dyar y Knab, 1906.

Capturas. Estancita (199'55.22” N 7040'14.07” O, 505 m s.n.m.), 12-I-2018, tres larvas; Pinar Dorado (197'9.78" N 70³7'56.06”' O, 547 m s.n.m.), 25-I-2018, 25 larvas; Pinar Dorado (197'9.78” N 70³7'56.06” O, 547 m s.n.m.), 07-II-2018, 11 larvas; La Confluencia (198'26.48” N 70³7'50.99” O, 497 m s.n.m.), 09-II-2018, tres larvas.

Hábitat. Cría en agua depositada en ambientes fitotélmicos, especialmente en las axilas de las brácteas de bromelias (Bromeliaceae). En Jarabacoa, sus larvas fueron capturadas en zonas rurales, semiurbanas y urbanas, en ambientes fitotélmicos junto a Wyeomyia mitchellii (Theobald, 1905), Ae. albonotatus, Ae. aegypti y Cx. biscaynensis.

Importancia médica. No se le conoce implicación en la transmisión de entidades infecciosas, a pesar de producir considerables molestias, ya que es una especie que se alimenta activamente de la sangre del ser humano y de la de otros animales. 
Identificación. Posee un índice sifónico muy elevado, alrededor de 7.0 y 8.0. Las sedas 4-X tienen menos de seis ramas (González Broche, 2006).

De las 22 especies identificadas en los últimos años en el municipio, ocho han sido reportadas en la literatura científica como vectores de patógenos al humano: An. albimanus, An. crucians, Cx. nigripalpus, Cx. quinquefasciatus, Ae. aeypti, Ae. albopictus, Ae. scapularis y Ps. confinnis. Otras, a pesar de no suponer un riesgo sanitario a priori, pueden producir considerables molestias a las personas producto de sus picaduras, como Ae. albonotatus o Wy. vanduzeei.

En relación a los vectores sinantrópicos, como Ae. aegypti, Ae albopictus o $C x$. quinquefasciatus, se recomienda eliminar y hacer inaccesibles la mayor cantidad de criaderos potenciales dentro del ámbito doméstico (Rodríguez Sosa et al., 2019b; Diéguez Fernández et al., 2019), así como realizar una limpieza exhaustiva en puntos críticos como los terrenos baldíos y los cementerios (González et al., 2019), a fin de reducir los riesgos sanitarios relacionados con la presencia de estos mosquitos. También es recomendable la práctica de la reducción, reutilización y reciclaje de los residuos sólidos urbanos, frecuentemente utilizados por estas especies de características oportunistas (Borge de Prada et al., 2018). Por lo que respecta a los vectores más agrestes, como An. albimanus, An. crucians, Ae. scapularis o Ps. confinnis, se recomienda la vigilancia periódica de ambientes lagunares permanentes y/o temporales ya que, a pesar de encontrarse alejados del entorno urbano, pueden suponer igualmente un peligro sanitario.

En República Dominicana, la repercusión sanitaria ocasionada por estos artrópodos durante los últimos años ha sido notable. Solo en 2019, la epidemia de dengue provocó más de 50 fallecidos y alrededor de 20000 casos notificados, mientras que el repunte de la malaria supuso la muerte de al menos tres personas y alrededor de 1200 casos (Ministerio de Salud Pública [MSP], 2019). Unido a lo anterior, no se debe subestimar la importancia de enfermedades virales como el chikungunya o el Zika, entre otras (Alarcón-Elbal et al., 2017), así como de la filariasis linfática, parasitosis altamente incapacitante también conocida como elefantiasis que se encuentra en vías de erradicación en la isla. Por tal motivo, es indispensable reforzar las medidas preventivas con campañas de sensibilización sostenidas en el tiempo y destinadas a informar, sensibilizar y movilizar a la sociedad para eliminar los riesgos asociados a la presencia de estos insectos en nuestras comunidades (Vásquez Bautista et al., 2019).

Toda esta información debe ser tenida en cuenta por los funcionarios de salud pública y los especialistas en control de vectores para la implementación de los correspondientes programas de vigilancia y control de mosquitos en Jarabacoa.

\section{CONCLUSIONES}

Se reporta la especie $C x$. interrogator por primera vez en la Hispaniola; además, se capturan otras especies no citadas con anterioridad en el municipio de Jarabacoa: An. crucians, Ae. scapularis, Ps. confinnis, Ps. infinis y Wy. vanduzeei. De las 22 especies catalogadas en Jarabacoa, ocho han sido fehacientemente reportadas como vectores de patógenos al ser humano; algunas se encuentran vinculadas mayormente al entorno rural, a pesar de lo cual también deben ser tenidas en cuenta dentro de los programas de vigilancia y control integral. 


\section{AGRADECIMIENTOS}

Los resultados del presente documento se derivan del proyecto "Sistema integrado de educación y vigilancia entomológica para la prevención y el control de enfermedades vehiculizadas por mosquitos (Diptera: Culicidae) en dos polos turísticos de República Dominicana", Proyecto No. 2015-112-145, subvencionado por el Fondo Nacional de Innovación y Desarrollo Científico y Tecnológico (FONDOCyT), Ministerio de Educación Superior, Ciencia y Tecnología (MESCyT). Asimismo, los autores desean agradecer encarecidamente a los administradores del Rancho Baiguate, por su implicación oficial en el proyecto.

\section{LITERATURA CITADA}

Alarcón-Elbal, P. M., R. Paulino-Ramírez, L. Diéguez-Fernández, R. Fimia-Duarte, K. A. Guerrero, y M. González. 2017. Arbovirosis transmitidas por mosquitos (Diptera: Culicidae) en la República Dominicana: una revisión. The Biologist, 15 (1): 193-219.

Arnell, J. H. 1976. Mosquito studies (Diptera, Culicidae). XXXIII - A revision of the Scapularis Group of Aedes (Ochlerotatus). Contribution of the American Entomological Institute, 13: $1-144$.

Belkin, J. N. y S. Heineman. 1973. Collection records of the project "Mosquitoes of Middle America”, Introduction: Dominican Republic. Mosquito Systematics, 5: 201-222.

Belkin, J. N., S. J. Heinemann y W. A. Page. 1970. The Culicidae of Jamaica (Mosquito studies. XXI). Contributions of the American Entomological Institute, 6 (1): 1-458.

Borge de Prada, M., M. A. Rodríguez-Sosa, Y. E. Vásquez Bautista, K. A. Guerrero y P. M. Alarcón-Elbal. 2018. Mosquitos (Diptera, Culicidae) de importancia médica asociados a residuos sólidos urbanos en Jarabacoa, República Dominicana. Revista Salud Jalisco, Número Especial, 20-27.

Carpenter, S. L. y J. LaCasse. 1955. Mosquitoes of North America (North of Mexico). University of California Press, Berkeley \& Los Angeles, 369 pp.

Cruz Francisco, V., D. I. Veda Moreno y A. Valdés Murillo. 2012. Aspectos ecológicos de la incidencia larval de mosquitos (Diptera Culicidae) en Tuxpan, Veracruz, México. Revista Colombiana de Entomología, 38 (1): 128-33.

Darsie, R. F. y R. A. Ward. 2005. Identification and geographical distribution of the Mosquitoes of North America, North of Mexico. $2^{\text {nd }}$ Edition. University of Florida Press. Gainesville, Florida, 383 pp.

Diéguez Fernández, L., M. Borge de Prada, M. A. Rodríguez Sosa, Y. E. Vásquez Bautista y P. M. Alarcón-Elbal. 2019. Un acercamiento al conocimiento de los hábitats larvarios de Aedes (Stegomyia) aegypti (Diptera: Culicidae) en el entorno doméstico en Jarabacoa, República Dominicana. Revista Cubana de Medicina Tropical, 71 (3): e386.

Elizondo-Quiroga, A. E. 2002. Taxonomía y distribución de los mosquitos (Diptera: Culicidae) de las regiones fisiográficas llanura costera del golfo y Sierra Madre Oriental del estado de Nuevo León, México. Universidad Autónoma de Nuevo León. Facultad de CC. BB, Nuevo León, 131 pp. 
Faran, M. E. 1980. A revision of the Albimanus Section of the subgenus Nyssorhynchus of Anopheles. Contributions of the American Entomological Institute, 15 (7): 1-216.

Forattini, O. P., I. Kakitani y M. A. M. Sallum. 1997. Breeding places of Aedes scapularis (Diptera: Culicidae) in artificial containers. Revista Saude Publica, 31: 519-22.

Gaffigan, T. V., R. C. Wilkerson, J. E. Pecor, J. A. Stoffer y T. Anderson. 2015. Systematic Catalog of Culicidae. Walter Reed Biosystematics Unit. Disponible en: http://www. mosquitocatalog.org/intro.aspx.

González, M. A., M. A. Rodríguez Sosa, Y. E. Vásquez Bautista, L. Diéguez Fernández, M. Borge de Prada, K. Guerrero y P. M. Alarcón-Elbal. 2019. Micro-environmental features associated to container-dwelling mosquitoes (Diptera: Culicidae) in an urban cemetery of the Dominican Republic. Revista de Biología Tropical, 67 (1): 132-145.

González Broche, R. 2006. Culícidos de Cuba. Editorial Científico Técnica. La Habana, 184 pp.

Klein, T. A., J. B. Lima y A. T. Tang. 1992. Seasonal distribution and diel biting patterns of culicine mosquitoes in Costa Marques, Rondônia, Brazil. Memórias do Instituto Oswaldo Cruz, 87 (1): 141-148.

Lourenço-de-Oliveira, R. y L. M. Deane. 1995. Presumed Dirofilaria immitis infections in wild-caught Aedes taeniorhynchus and Aedes scapularis in Rio de Janeiro, Brazil. Memórias do Instituto Oswaldo Cruz, 90 (3): 387-388.

Marchi, M. J., P. A. Pereira, R. M. de Menezes y R. M. Tubaki. 2012. New records of mosquitoes carrying Dermatobia hominis eggs in the state of São Paulo, southeastern Brazil. Journal of the American Mosquito Control Association, 28 (2): 116-118.

Marquetti Fernández, M. C., Y. S. Jean, C. A. Fuster Callaba y L. Somarriba López. 2012. The first report of Aedes (Stegomyia) albopictus in Haiti. Memórias do Instituto Oswaldo Cruz, 107 (2): 279-281.

Mayne, B. 1919. The occurrence of malaria parasites in Anopheles crucians in nature: percentage of infection of Anopheles quadrimaculatus and latest date found infected in Northern Louisiana. Public Health Reports (1896-1970), 34 (25): 1355-1357.

Ministerio de Salud Pública / Dirección General de Epidemiología (MSP/DIGEPI). 2019. Semana Epidemiológica (SE) No. 50. Disponible en: http://digepisalud.gob.do/docs/Boletines\%20 epidemiologicos/Boletines\%20semanales/2019/Boletin\%20Semanal\%2050-2019.pdf.

Mitchell, C. J. y O. P. Forattini. 1984. Experimental transmission of Rocio encephalitis virus by Aedes scapularis (Diptera: Culicidae) from the epidemic zone in Brazil. Journal of Medical Entomology, 21 (1): 34-37.

Mitchell, C. J., T. P. Monath, M. S. Sabattini, C. B. Cropp, J. F. Daffner, C. H. Calisher, W. L. Jakob y H. A. Christensen. 1985. Arbovirus investigations in Argentina, 1977-1980. II. Arthropod collections and virus isolations from Argentine mosquitoes. The American Journal of Tropical Medicine and Hygiene, 34 (5): 945-955.

Orgaz Agüera, F. y P. Cañero - Morales. 2015. El ecoturismo como motor de desarrollo en zonas rurales: un estudio de caso en República Dominicana. Ciencia y Sociedad, 40 (1): 47-76. 
Ortega-Morales, A., P. Mis-Ávila, A. Elizondo-Quiroga, R. E. Harbach, Q. K. Siller-Rodríguez y I. Fernández-Salas. 2010. Themosquitoes of Quintana Roo State, Mexico (Diptera: Culicidae). Acta Zoológica Mexicana (n.s.), 26 (1): 33-46.

Ortiz, D. I., M. Anishchenko y S. C. Weaver. 2005. Susceptibility of Psorophora confinnis (Diptera: Culicidae) to infection with epizootic (subtype IC) and enzootic (subtype ID) Venezuelan Equine encephalitis viruses. Journal of Medical Entomology, 42 (5): 857-863.

Pauvolid-Corrêa, A., J. L. Kenney, D. Couto-Lima, Z. M. S. Campos, H. G. Schatzmayr, R. M. R. Nogueira, A. C. Brault y N. Komar. 2013. Ilheus virus isolation in the Pantanal, west-central Brazil. PLoS Negleted Tropical Diseases, 7: 1-8.

Peña, C. J. y D. D. Chadee. 2004. Mansonia dyari, Mansonia flaveola and Aedeomyia squamipennis in the Dominican Republic, three new country records. Journal of the American Mosquito Control Association, 20: 449-450.

Pérez Menzies, M., Y. Cutiño-Alba, Y. Cid-Acosta, G. Torres-Guayanes, R. M. CastilloQuesada, Y. Alfonso-Herrera, R. González-Broche y M. C. Marquetti-Fernández. 2018. Presencia larval de Culex (Culex) interrogator (Dyar \& Knab) (Diptera: Culicidae) en Cuba. Revista Cubana de Medicina Tropical, 70 (3): 108-113.

Rachou, R. G., M. M. Lima, J. A. F. Neto y C. M. Martins. 1995. Inquérito epidemiológico de filariose bancroftiana em uma localidade de Santa Catarina. Como fase preliminar de uma prova profilática. Constatação de transmissão extradomiciliária por um novo vetor, Aedes scapularis. Revista Brasileira de Malariologia e Doenças Tropicais, 7: 51-70.

Rodríguez Sosa, M. A., J. Rueda, Y. E. Vásquez Bautista, R. Fimia-Duarte, M. Borge de Prada, K. A. Guerrero y P. M. Alarcón-Elbal. 2019a. Diversidad de mosquitos (Diptera: Culicidae) de Jarabacoa, República Dominicana. Graellsia, 75: e084.

Rodríguez Sosa, M. A., L. Diéguez Fernández, M. Borge de Prada, Y. E. Vásquez Bautista y P. M. Alarcón-Elbal. 2019b. Sitios de cría de Aedes albopictus (Skuse) (Diptera: Culicidae) en el entorno doméstico en Jarabacoa, República Dominicana. Revista Chilena de Entomología, 45: 403-410.

Rodríguez-Sosa, M. A., Y. E. Vásquez-Bautista, R. Fimia-Duarte, K. A. Guerrero y P. M. Alarcón-Elbal. 2018. Primer registro de Uranotaenia cooki (Diptera: Culicidae) para República Dominicana. Revista Colombiana de Entomología, 44 (1): 141-144.

Rueda, J., M. A. Rodríguez Sosa, Y. E. Vásquez Bautista, K. A. Guerrero y P. M. Alarcón-Elbal. 2018. Primera cita de Culex (Culex) garciai González-Broche, 2000 (Diptera: Culicidae) para La Española. Anales de Biología, 40: 95-101.

Shin, D., G. F. O’Meara, A. Civana, D. A. Shroyer y E. Miqueli. 2016. Culex interrogator (Diptera: Culicidae), a mosquito species new to Florida. Journal of Vector Ecology, 41 (2): 316-319.

Spence, L., C. R. Anderson, T. H. G. Aitken y W. G. Downs. 1962. Melao virus, a new agent isolated from Trinidadian mosquitoes. American Journal of Tropical Medicine and Hygiene, 11: 687-690. 
Unlu, I., W. L. Kramer, A. F. Roy y L. D. Foil. 2010. Detection of West Nile Virus RNA in Mosquitoes and Identification of Mosquito Blood Meals Collected at Alligator Farms in Louisiana. Journal of Medical Entomology, 47 (4): 625-633.

Vásquez Bautista, Y. E., Y. Hernández Barrios, M. A. Rodríguez-Sosa, E. del Carmen Rosario, J. C. Durán Tiburcio y P. M. Alarcón-Elbal. 2019. «Sácale los pies al mosquito»: resultados parciales de la implementación de un programa educativo en República Dominicana. Ciencia y Sociedad, 44: 33-49.

WRBU, 2020. Walter Reed Biosystematics Unit: Systematic catalog of Culicidae. Walter Reed Biosystematics Unit, Smithsonian Institution, Washington D.C. Disponible en: http://www. mosquitocatalog.org/.

Zapata-Peniche, A., P. Manrique-Saide, E. A. Rebollar-Téllez, A. Che-Mendoza y F. Dzul-Manzanilla. 2007. Identificación de larvas de mosquitos (Diptera: Culicidae) de Mérida, Yucatán, México y sus principales criaderos. Revista Biomédica, 18: 3-17.

[Recibido: 12 de enero, 2020. Aceptado para publicación: 26 de abril, 2020] 